\title{
ESTRESSE NO COTIDIANO DE GRADUANDOS DE ENFERMAGEM DE UM INSTITUTO FEDERAL DE ENSINO
}

\section{STRESS IN THE DAILY LIFE OF NURSING GRADUATION STUDENTS FROM A FEDERAL INSTITUTE OF EDUCATION}

\section{ESTRÉS EN EL COTIDIANO DE GRADUANDOS DE ENFERMERÍA DE UN INSTITUTO FEDERAL DE ENSEÑANZA}

Leni de Lima Santana ${ }^{1}$, Walkiria Denipotti Beljaki² ${ }^{2}$ Mariângela Gobatto ${ }^{3}$, Rafael Haeffner ${ }^{4}$, Milena Hohmann Antonacci ${ }^{5}$, Jane Aparecida Pereira Buzzi ${ }^{6}$.

\section{RESUMO}

Objetivos: identificar os níveis de estresse e caracterizar a sintomatologia entre discentes de graduação em enfermagem de um Instituto Federal de Ensino. Método: Estudo descritivo transversal cuja coleta de dados ocorreu por meio da aplicação do Inventário de Sintomas de Estresse de Lipp e de um questionário sociodemográfico elaborado pelos pesquisadores. Os resultados foram analisados por meio de estatística descritiva. Resultados: A análise evidenciou que $73,7 \%$ dos alunos apresentavam algum nível de estresse, com destaque para sintomas na fase de resistência. Os sintomas predominaram entre acadêmicos autodeclarados pardos $(80,0 \%)$, provenientes de escolas públicas $(74,3 \%)$, não cotistas $(74,1 \%)$, repetentes $(83,3 \%)$ e entre acadêmicos que não trabalham formalmente $(73,8 \%)$. O desfecho foi associado às variáveis independentes: tipo de cota $(p=$ $0,019)$, período letivo $(p=0,003)$ e uso de medicamentos $(p=0,04)$. Quanto à sintomatologia apresentada, predominaram os sintomas de caráter psicológico, apresentados por $50 \%$ da amostra. Conclusão: todos os períodos do curso avaliado apresentaram estresse em nível variado o que merece a atenção por parte da instituição e do corpo docente no sentido de rever as metodologias adotadas, bem como propor ações que visem o bem-estar e o desempenho acadêmico.

Descritores: Enfermagem; Estudantes de Enfermagem; Estresse Psicológico; Saúde Mental.

\section{ABSTRACT}

Objective: to identify stress levels and to characterize the symptomatology among nursing undergraduate students of a Federal Institute of Education. Method: A cross-sectional descriptive study whose data collection was carried out through the application of the Lipp Stress Symptom Inventory and a sociodemographic questionnaire prepared by the researchers. The results were analyzed using descriptive statistics. Results: The analysis showed that $73.7 \%$ of the students presented some level of stress, with emphasis on symptoms in the resistance phase. The symptoms predominated among self-reported brown students (80.0\%), from public schools $(74.3 \%)$, non-quota students $(74.1 \%)$, repeaters $(83.3 \%)$ and non-formal academics $(73.8 \%)$. The outcome was associated with the independent variables: type of quota $(p=0.019)$, period $(p=0.003)$ and medication use $(p=0.04)$. As for the symptomatology presented, the symptoms of a psychological nature, presented by $50 \%$ of the sample, predominated. Conclusion: all the periods of the evaluated course presented stress at a varied level, which deserves the attention of the institution and the faculty in order to review the adopted methodologies, as well as to propose actions that aim at the well-being and the academic performance.

Keywords: Nursing; Nursing students; Psychological stress; Mental health.

\section{RESUMEN}

Objetivo: identificar los niveles de estrés y caracterizar las sintomatologías entre discentes de Graduación en Enfermería de un Instituto Federal de Enseñanza. Método: Estudio descriptivo transversal cuyos datos fueron recolectados por medio del Inventario de Síntomas de Estrés de Lipp y de un cuestionario sociodemográfico elaborado por los investigadores. Los resultados fueron analizados por medio de estadística descriptiva. Resultados: El análisis evidenció que el 73,7\% de los alumnos presentan algún nivel de estrés, con atención para los síntomas en la fase de resistencia. Los síntomas predominaron entre académicos pardos $(80,0 \%)$, provenientes de escuela pública $(74,3 \%)$, no cotizantes $(74,1 \%)$, alumnos repitientes $(83,3 \%)$ y entre académicos que no trabajan formalmente $(73,8 \%)$. El resultado fue asociado a las variables independientes: tipo de cuota $(p=0,019)$, período lectivo $(p=0,003)$ y uso de medicamentos $(p=0,04)$. En cuanto a la sintomatología presentada, predominaron los síntomas de carácter psicológico, presentados por el $50 \%$ de la muestra. Conclusión: todos los períodos del curso evaluado presentaron estrés a nivel variado lo que merece la atención por parte de la institución y del cuerpo docente en el sentido de revisar las metodologías adoptadas, así como proponer acciones que visen el bienestar y el desempeño académico.

Descriptores: Enfermería; Estudiantes de Enfermería; Estrés Psicológico; Salud mental.

${ }^{1}$ Graduada em Enfermagem. Doutoranda em Enfermagem pela Universidade Federal do Paraná. Docente no Instituto Federal do Paraná. ${ }^{2}$ Graduada em Enfermagem pelo Instituto Federal do Paraná. ${ }^{3}$ Graduada em Enfermagem. Mestre em Enfermagem pela Universidade Federal de Santa Maria. Docente no Instituto Federal do Paraná. ${ }^{4}$ Graduado em Enfermagem. Doutorando em Epidemiologia pela Universidade de São Paulo. ${ }^{5}$ Graduada em Enfermagem. Doutora em Enfermagem Psiquiátrica pela Universidade de São Paulo. Docente no Instituto Federal do Paraná. ${ }^{6}$ Graduada em Psicologia pela Pontifícia Universidade Católica do Paraná. DOI: http://dx.doi.org/10.19175/recom.v8i0.2738 


\section{INTRODUÇÃO}

A competitividade do mercado de trabalho tem exigido do ser humano cada vez mais habilidade, competência e produtividade. Aliado a isso, as exigências pessoais por realização familiar, social e profissional requerem cada vez mais a capacidade de superação do indivíduo. Contudo, quando as exigências diárias ultrapassam a capacidade de reação e adaptação do organismo, inicia-se um processo de desgaste físico e psicológico caracterizado, entre outros fatores, pelos sintomas de estresse ${ }^{(1)}$.

Por envolver reações do organismo frente às situações difíceis ou excitantes, o estresse pode ocorrer em qualquer pessoa, independentemente de idade, sexo e situação socioeconômica ${ }^{(1)}$.

No universo acadêmico, níveis elevados de estresse têm sido evidenciados entre discentes de diversas áreas de formação(2). 0 acúmulo de tarefas, as cobranças, os problemas institucionais, as exigências da vida pessoal e social, as expectativas e preocupações com o mundo do trabalho e o relacionamento interpessoal entre os alunos $^{(3)}$ são alguns fatores associados à sua ocorrência.

$\mathrm{Na}$ enfermagem, fatores como as exigências por uma formação generalista, humanista, crítica e reflexiva exigem do acadêmico uma visão holística e tomadas de decisão importantes no cuidado ao paciente ${ }^{(4)}$. Além desses aspectos, o fato de a enfermagem ser responsável pelo cuidado ao sujeito fragilizado por uma patologia, ou pelo cuidado preventivo, o que exige grande responsabilidade, pode contribuir para a ocorrência de estresse nessa população.

Conceitualmente, estresse trata-se do resultado inespecífico de qualquer demanda sobre o corpo, seja ele de efeito somático ou mental. O seu desencadeamento depende de um agente e sua manifestação pode ocorrer em distintas fases: alerta, resistência, quase exaustão e exaustão ${ }^{(1,5)}$.

A fase de alerta corresponde à ativação do sistema nervoso simpático frente a uma ameaça externa como uma forma de preparar o corpo para o enfrentamento do perigo. A fase de resistência corresponde ao esforço de adaptação frente a um estressor de longa duração ou de intensidade exacerbada, o que torna o indivíduo mais vulnerável às doenças ${ }^{(5)}$.
Quando o organismo experimenta uma sensação de esgotamento, aumentando a chance da perda de controle emocional, tem-se a fase de quase exaustão(1) e quando, finalmente, a resistência da pessoa não é suficiente para lidar com a fonte de estresse, ou quando outros estressores ocorrem simultaneamente, o estado evolui para a fase de exaustão, cuja manifestação pode se dar por meio de sintomas físicos ou psicológicos e culminar com o aparecimento de doenças ${ }^{(5)}$.

Os sintomas físicos podem se manifestar por meio de sinais e alterações gastrointestinais; problemas cardiovasculares, tais como taquicardia, hipertensão arterial, formigamento em membros superiores e inferiores; problemas dermatológicos, mal-estar geral; entre outros. As manifestações psicológicas se caracterizam principalmente por alterações de humor, depressão, angústia, ansiedade, irritabilidade, apatia, tiques, problemas de relacionamento, distúrbios do sono e dificuldades de concentração(6).

No meio acadêmico, os níveis de estresse podem afetar negativamente a qualidade de vida dos discentes, interferir no esquema de estudo e tornar-se um obstáculo tanto para o desempenho estudantil quanto profissional ${ }^{(7)}$.

Assim, considerando que a identificação do estresse no ambiente acadêmico contribui para a prevenção de agravos e promoção da saúde, principalmente mental, traçaram-se, como objetivos deste estudo: identificar os níveis de estresse e caracterizar a sua sintomatologia entre discentes de graduação em enfermagem de um Instituto Federal de Ensino.

\section{METODO}

Estudo quantitativo, transversal e descritivo, realizado em um Campus do Instituto Federal do Paraná (IFPR), instituição voltada à educação superior, básica e profissional, especializada na oferta gratuita de educação profissional e tecnológica nas diferentes modalidades e níveis de ensino. O cenário do estudo atende, atualmente, cerca de dois mil alunos em treze cursos superiores e dois cursos técnicos.

O curso de enfermagem é anual e estruturado em 10 semestres. No período da coleta de dados, outubro de 2016, havia 95 alunos matriculados, sendo 25 no segundo período, 20 no quarto, 15 no sexto, 19 no oitavo e 16 no décimo período. Foram excluídos do 
estudo, os discentes ausentes das atividades acadêmicas na data da coleta de dados, independentemente dos motivos, e a aluna envolvida no estudo, sendo assim, a amostra foi composta por 80 alunos.

Para a caracterização dos sujeitos, foi aplicado um questionário elaborado pelos pesquisadores contendo variáveis de identificação pessoal, residencial, acadêmicas e trabalhistas. Para a avaliação do nível de estresse, foi utilizado o Inventário de Sintomas de Estresse de Lipp (ISSL), validado para sujeitos acima de 15 anos $^{(5)}$.

O instrumento ISSL é composto por uma lista de sintomas físicos e psicológicos, que permite diagnosticar se a pessoa está sob estresse, em que fase do processo se encontra (alerta, resistência, quase exaustão e exaustão) e se sua sintomatologia é mais típica da área somática ou cognitiva. Metodologicamente, os sintomas são agrupados em quatro quadros, sendo o primeiro composto por 15 itens que correspondem à fase de alerta. O segundo quadro, também composto por 15 itens, é dividido em duas partes, sendo que os escores entre 4 e nove correspondem à fase de resistência e os escores entre 10 e 15, à fase de quase exaustão. Já o terceiro quadro apresenta 23 itens que correspondem à fase de exaustão, totalizando, assim, 53 itens avaliados ${ }^{(6)}$.

0 estabelecimento do diagnóstico de estresse é feito se qualquer dos escores brutos atingirem os limites determinados. A fase do estresse é determinada por porcentagens préestabelecidas, de acordo com os valores brutos dos sintomas. A maior porcentagem obtida indica a fase do estresse em que a pessoa se encontra. Caso haja empate entre as porcentagens, o diagnóstico é feito pela fase mais avançada ${ }^{(6)}$.

Para caracterização de sintomatologia predominante, considerou-se apenas a fase de estresse na qual o indivíduo se encontrava e compararam-se as porcentagens obtidas nos sintomas físicos com as porcentagens dos sintomas psicológicos, conforme determina 0 instrumento. A sintomatologia que obteve a maior porcentagem revelou se a manifestação do estresse era fisiológica ou psicológica ${ }^{(6)}$.

Os instrumentos foram aplicados em sala de aula com consentimento dos discentes e dos docentes e após autorização da coordenação do curso. A aplicação se deu de forma individual, em espaço coletivo de sala de aula, e os instrumentos recolhidos ao término do preenchimento do inventário, que teve duração aproximada de 15 minutos.

As variáveis analisadas foram do tipo sociodemográficas (sexo, idade, raça/etnia, estado civil, convívio pessoal, possui filhos, localidade de residência, tempo de deslocamento até o curso); estudantis específicas (cotas, tipo de cotas, período letivo, repetência, tipo de custeio para o ensino médio); trabalho (sim/não); e uso de medicamentos (sim/não).

A análise descritiva dos dados foi composta por frequências absolutas e relativas para as variáveis categóricas. Para a variável idade foram utilizadas medidas de tendências centrais e dispersão (média, mediana, desvio padrão e valor mínimo e máximo) e categorização em tercil para obtenção da faixa etária ( $\leq 20$ anos, $21-22$ anos, $\geq 23$ anos).

Para a análise de inferência estatística, foi utilizado o teste exato de Fisher a fim de verificar as diferenças de proporções entre as categorias em análise de cada variável independente e a variável dependente (fases do estresse), sendo considerado significativo $p<0,05$.

Este estudo é parte do projeto "Estresse no cotidiano acadêmico: com a voz discentes e docentes de um Instituto Federal de Ensino" aprovado pelo CAAE: 60009916.0.0000.5573 sob no. 1.850.689, obedecendo aos preceitos éticos. Todos os participantes receberam esclarecimentos e concordaram em participar voluntariamente do estudo mediante assinatura do Termo de Consentimento Livre e Esclarecido.

\section{RESULTADOS E DISCUSSÃO}

Participaram da pesquisa 80 estudantes do curso de enfermagem, com predomínio do sexo feminino (90\%). A média de idade dos participantes do estudo foi de 22,9 anos, mediana de 21 anos, desvio padrão de 6,3 anos, idade mínima de 17 e máxima de 46 anos, com destaque para faixa etária até 20 anos $(42,5 \%)$, solteiro/divorciado $(75,0 \%)$, sem filhos $(77,5 \%)$, convívio com familiares $(62,5 \%)$, do município sede do curso $(92,5 \%)$, com tempo médio de deslocamento casa/instituição de 15 a 30 minutos $(58,7 \%)$, e que não exerciam atividade remunerada na ocasião do estudo $(81,2 \%)$.

Esses resultados assemelham-se aos obtidos em estudo desenvolvido na Universidade Federal do Piauí, em que se constatou que $77,7 \%$ dos acadêmicos eram do sexo feminino, $51,9 \%$ com média de 22 anos de idade, 85,9\% só 
estudavam; $88,8 \%$ eram solteiras e $89,3 \%$ não tinham filhos ${ }^{(8)}$.

Resultados semelhantes foram evidenciados em estudo desenvolvido em uma Universidade pública de João Pessoa o qual teve como objetivo estimar o nível de estresse em acadêmicos de enfermagem. Os autores evidenciaram que $90 \%$ dos alunos entrevistados era do sexo feminino, a média da idade era de 22,74 anos e apenas 8,6\% deles desempenhavam as funções de aluno e profissional ${ }^{(9)}$.

O fato de $37,5 \%$ dos acadêmicos terem declarado que não residem com familiares pode estar relacionada à localização geográfica da instituição, distante de centros urbanos, o que contribui para o fato de acadêmicos de diferentes municípios do Estado e de Estados próximos residirem em pensionatos, casas de aluguel ou com amigos. O fato de residir longe da família faz com que esses acadêmicos assumam novas responsabilidades para as quais podem não estar preparados.

Infere-se que a prevalência do sexo feminino se deva ao fato de a profissão estar histórica e culturalmente ligada às práticas ligadas à mulher. A enfermagem tem como objeto o cuidado, que sempre foi visto como uma qualidade feminina ${ }^{(8)}$. Ela é preparada desde pequena para ser mãe, cuidar da casa, da família, dos doentes, entre outros afazeres domésticos. Atualmente, a sociedade exige das mulheres, de maneira geral, uma sobrecarga de tarefas, tendo em vista que precisam conciliar as atividades domésticas com a carreira profissional e com as exigências sociais ${ }^{(10)}$.

A predominância de acadêmicos que não conciliam trabalho e estudo pode estar relacionada a dois fatores. Primeiro, pelo fato de o curso de Enfermagem na instituição estudada ter carga horária em período integral, o que reduz a possibilidade de o aluno exercer algum trabalho remunerado e ainda pela grande procura pelo curso por jovens recém-saídos do ensino fundamental, motivados pelas facilidades no ingresso em instituições públicas de ensino superior, bem como em busca de uma profissão que julgam com maior inserção ocupacional.

A maior ocorrência de solteiros reflete a realidade do curso, composto, em sua maioria, por mulheres jovens, o que vem ao encontro com a realidade brasileira. Nas últimas décadas as mulheres têm optado por se casar mais tarde $\mathrm{e}^{(11)}$, dando prioridade à sua formação profissional e inserção no mercado de trabalho ${ }^{(8)}$.

Quanto a outras variáveis, as categorias que prevaleceram foram: autodeclarados brancos $(73,7 \%)$, não entrar por sistema de cotas $(72,5 \%)$, proveniente do ensino público $(92,5 \%)$, não ter repetido período letivo (85\%) e não usar medicamentos (76,2\%).

As características étnico-raciais condizem com a população do Sul do país, cuja população branca representa $76,0 \%$ do total ${ }^{(12)}$. Embora o número de alunos que não aderiram ao curso por meio de cotas seja representativo, ressalta-se que a Instituição em que o estudo foi desenvolvido possui política de ações afirmativas e cumpre com o estabelecido pela legislação no que se refere ao número de vagas destinadas para cotistas $^{(13)}$.

Sobre a ocorrência de estresse na população estudada, observou-se que $59(73,7 \%)$ alunos foram classificados com estresse em alguma fase. No que se refere às variáveis sociodemográficas, observou-se a ocorrência de estresse em $100 \%$ da população masculina, maior frequência dos sintomas entre os alunos com idade entre 21 e 22 anos, que se declararam pardos $(80,0 \%)$, solteiros ou divorciados $(75,0 \%)$, com filhos $(77,8 \%)$, que residem sozinhos ou com outros que não familiares ou amigos $(85,7 \%)$ e residentes no município onde estudam $(74,3 \%)$, com destaque para a fase de resistência, conforme Tabela 1.

Tabela 1 - Níveis de estresse em discentes de enfermagem de um Instituto Federal de Ensino, segundo ISSL. Brasil, out. 2016.

\begin{tabular}{lcccccc}
\hline \multicolumn{1}{c}{ Variável } & \multicolumn{2}{c}{ Fases do Estresse } \\
& Sem estresse & Resistência & Quase exaustão & Exaustão & & \\
Sexo & $\mathbf{N}(\%)$ & $\mathbf{N ~ ( \% )}$ & $\mathbf{N}(\%)$ & $\mathbf{N}(\%)$ & Total N (\%) & Valor de $\mathbf{p}^{*}$ \\
Feminino & & & & & \\
Masculino & $21(29,17)$ & $37(51,39)$ & $11(15,28)$ & $3(4,17)$ & $72(100,0)$ & \\
Total & $0(0,0)$ & $6(75,0)$ & $1(12,5)$ & $1(12,5)$ & $8(100,0)$ & \\
\hline
\end{tabular}




\begin{tabular}{|c|c|c|c|c|c|c|}
\hline \multirow{3}{*}{ Variável } & \multicolumn{4}{|c|}{ Fases do Estresse } & & \multirow[b]{3}{*}{ Valor de $\mathrm{p}^{*}$} \\
\hline & Sem estresse & Resistência & Quase exaustão & Exaustão & & \\
\hline & $\mathrm{N}(\%)$ & $N(\%)$ & $\mathrm{N}(\%)$ & N (\%) & Total N (\%) & \\
\hline Faixa etária & & & & & & $=0,941$ \\
\hline$\leq 20$ anos & $11(32,35)$ & $16(47,06)$ & $5(14,71)$ & $2(5,88)$ & $34(100,0)$ & \\
\hline $21-22$ anos & $4(18,18)$ & $13(59,09)$ & $4(18,18)$ & $1(4,55)$ & $22(100,0)$ & \\
\hline$\geq 23$ anos & $6(25,0)$ & $14(58,33)$ & $3(12,5)$ & $1(4,17)$ & $24(100,0)$ & \\
\hline Total & $21(26,25)$ & $43(53,75)$ & $12(15,0)$ & $4(5,0)$ & $80(100,0)$ & \\
\hline Raça/etnia & & & & & & $=0,340$ \\
\hline Negra & $0(0,0)$ & $1(50,0)$ & $1(50,0)$ & $0(0,0)$ & $2(100,0)$ & \\
\hline Branca & $15(25,42)$ & $34(57,63)$ & $7(11,86)$ & $3(5,08)$ & $59(100,0)$ & \\
\hline Parda & $3(20,0)$ & $7(46,67)$ & $4(26,67)$ & $1(6,67)$ & $15(100,0)$ & \\
\hline Indígena & $3(75,0)$ & $1(25,0)$ & $0(0,0)$ & $0(0,0)$ & $4(100,0)$ & \\
\hline Total & $21(26,25)$ & $43(53,75)$ & $12(15,0)$ & $4(5,0)$ & $80(100,0)$ & \\
\hline Estado Civil & & & & & & $=0,713$ \\
\hline Solteiro/divorciado & $15(25,0)$ & $31(51,67)$ & $10(16,67)$ & $4(6,67)$ & $60(100,0)$ & \\
\hline Casado/união & & & & & & \\
\hline estável & $6(30,0)$ & $12(60,0)$ & $2(10,0)$ & $0(0,0)$ & $20(100,0)$ & \\
\hline Total & $21(26,25)$ & $43(53,75)$ & $12(15,0)$ & $4(5,0)$ & $80(100,0)$ & \\
\hline Filhos & & & & & & $=0,703$ \\
\hline Não & $17(27,42)$ & $31(50,0)$ & $10(16,13)$ & $4(6,45)$ & $62(100,0)$ & \\
\hline Sim & $4(22,22)$ & $12(66,67)$ & $2(11,11)$ & $0(0,0)$ & $18(100,0)$ & \\
\hline Total & $21(26,25)$ & $43(53,75)$ & $12(15,0)$ & $4(5,0)$ & $80(100,0)$ & \\
\hline Convívio & & & & & & $=0,450$ \\
\hline Familiares & $16(32,0)$ & $25(50,0)$ & $8(16,0)$ & $1(2,0)$ & $50(100,0)$ & \\
\hline Amigos & $3(18,75)$ & $10(62,5)$ & $2(12,50$ & $2(6,25)$ & $16(100,0)$ & \\
\hline Sozinho/ & & & & & & \\
\hline Outros & $2(14,29)$ & $8(57,14)$ & $2(14,29)$ & $2(14,29)$ & $14(100,0)$ & \\
\hline Total & $21(26,25)$ & $43(53,75)$ & $12(15,0)$ & $4(5,0)$ & $80(100,0)$ & \\
\hline Município de residência & & & & & & $=0,290$ \\
\hline O mesmo em que & & & & & & \\
\hline estuda & $19(25,68)$ & $41(55,41)$ & $11(14,86)$ & $3(4,05)$ & $74(100,0)$ & \\
\hline Outros & $2(33,33)$ & $2(33,33)$ & $1(16,67)$ & $1(16,67)$ & $6(100,0)$ & \\
\hline Total & $21(26,25)$ & $43(53,75)$ & $12(15,0)$ & $4(5,0)$ & $80(100,0)$ & \\
\hline
\end{tabular}

*Teste exato de Fisher: $p<0,05$.

Sobre esse aspecto, os resultados evidenciados nesse estudo assemelham-se aos obtidos em pesquisa realizada em uma universidade pública federal em João Pessoa-PB, a qual objetivou estimar o nível de estresse em acadêmicos de enfermagem. Os autores evidenciaram que $49,7 \%$ dos alunos apresentaram estresse e desses, 42,4\% apresentavam-se na fase de resistência, sendo esta a de segundo maior predomínio, seguida das fases de quase exaustão (3,3\%), alerta $(2,7 \%)$ e exaustão $(1,3 \%)^{(9)}$. Em outro estudo, desenvolvido em Minas Gerais, $78 \%$ de todos os alunos avaliados apresentavam estresse ${ }^{(14)}$ e em graduandos de uma escola pública do Rio de Janeiro verificou-se que $1,6 \%$ do grupo total apresentava estresse na fase de alerta, $41,2 \% \mathrm{em}$ resistência, 5,2 \% na fase de quase-exaustão e $2,1 \%$ na fase de exaustão(3).

Esses resultados são preocupantes tanto pelos efeitos físicos e psicológicos à saúde dos acadêmicos quanto pelos riscos a que estão submetidos os pacientes sob seus cuidados. Em estado de estresse, os acadêmicos de enfermagem podem realizar práticas inseguras e protagonizar histórias de danos severos e irreversíveis tanto à sua saúde quanto à saúde daqueles que estão sob os seus cuidados uma vez que, nessas condições, podem ocorrer falhas de memória e redução na capacidade de concentração ${ }^{(15)}$.

Observou-se, ainda, a prevalência do estresse na fase de resistência em 53,7\% ( $n=43)$. Isso pode indicar que os acadêmicos conseguem se proteger até certo ponto dos estímulos estressores, visto que $15,0 \%(n=12)$ encontramse na fase de quase-exaustão e $5,0 \%(n=4)$ na fase de exaustão, fator que merece atenção devido às consequências que podem trazer à saúde física e psicológica dos mesmos. Ressaltase que essa fase se trata de um estágio transitório de caráter pré-patológico em que o organismo pode apresentar sinais de enfraquecimento, ficando mais suscetível às doenças de um modo geral $^{(6)}$. 
Nesse aspecto, destaca-se a importância de adequação das práticas pedagógicas, o repensar sobre a atuação docente, além da adequação das condições de trabalho aos docentes que convivem com o dilema de atender as exigências curriculares, as demandas dos campos de prática e os anseios, medos, dilemas e inseguranças acadêmicas. Ressalta-se que, em situações inadequadas de trabalho, na ausência de apoio institucional e quando o número de alunos ultrapassa a capacidade docente de supervisão, tem-se reduzido o tempo de atenção ao acadêmico e, consequentemente, elevado o grau de insegurança e de estresse nos alunos, o que pode se traduzir em um risco para os pacientes que estão sob os seus cuidados ${ }^{(15-16)}$.

Sobre o nível de estresse segundo o sexo dos participantes, observou-se uma proporção mais elevada do sexo masculino ao nível de exaustão, resultados que se assemelham aos evidenciados em pesquisa realizada com 480 acadêmicos, no estado do Paraná, para investigar a vulnerabilidade ao estresse em adultos jovens. No referido estudo, verificou-se que $47,9 \%$ dos homens e $36,1 \%$ das mulheres apresentaram alta vulnerabilidade ao estresse ${ }^{(17)}$. Ressalta-se que essa divergência nos resultados pode ser consequência do tamanho da amostra masculina neste estudo, não servindo, assim, de parâmetros de comparação.

Contudo, talvez seja um indicativo de que os acadêmicos do sexo masculino, ao defrontarse com situações desencadeantes de estresse, tenham menos recursos de adaptação, principalmente quando integram um grupo heterogêneo e marcado por divergências culturais e estilos de vida. De modo geral, estudantes do sexo feminino tendem a utilizar estratégias baseadas no suporte social, na emoção e em práticas religiosas que, embora possam se configurar como uma postura de esquiva e de negação frente à adaptação acadêmica ${ }^{(18)}$, podem auxiliar no enfrentamento dos fatores estressantes.

Os acadêmicos que moram em pensão ou sozinhos apresentaram-se em alguma fase de estresse, superando os resultados evidenciados entre os que moram com amigos ou com familiares. Esses resultados revelam a importância de um equilíbrio para conciliar as atividades acadêmicas com a vida familiar e social. Pressupõe-se que os alunos enfrentem dificuldades para conciliar as atividades estabelecidas na grade curricular com as exigências pessoais, emocionais e sociais, o que merece atenção da instituição ${ }^{(2-3,7,14)}$, uma vez que o suporte social atua como facilitador na adaptação acadêmica e contribui para o enfrentamento de comportamentos inseguros, ansiosos, passivos e dependentes, decorrentes da incapacidade individual de lidar com os estressores $^{(18)}$. O incentivo às visitas familiares pode ser uma forma alternativa de aliviar o sofrimento mental ${ }^{(14)}$.

No que se refere aos fatores acadêmicos, as variáveis independentes "tipo de cota" ( $p=$ $0,019)$ e "período letivo" ( $p=0,003)$ mostraramse associadas ao desfecho. A maior frequência de estresse foi evidenciada nos acadêmicos que cursaram o ensino médio em escolas públicas $(74,3 \%)$, não cotistas $(74,1 \%)$, matriculados no sexto e no quarto período $(99,9 \%$ e $93,3 \%$, respectivamente), que declararam repetência $(83,3 \%)$ e entre acadêmicos que não trabalham formalmente $(73,8 \%)$. Nessas variáveis, também houve predomínio de estresse na fase de resistência (Tabela 2).

Tabela 2 - Níveis de estresse em discentes de enfermagem de um Instituto Federal de Ensino, segundo variáveis acadêmicas, de acordo com o ISSL. Brasil, out. 2016.

\begin{tabular}{|c|c|c|c|c|c|c|}
\hline \multicolumn{7}{|c|}{ Fases do Estresse } \\
\hline Variável & & & Quase & & & \\
\hline & $\begin{array}{c}\text { Sem estresse } \\
\mathbf{N}(\%)\end{array}$ & $\begin{array}{c}\text { Resistência } \\
\text { N (\%) }\end{array}$ & $\begin{array}{c}\text { exaustão } \\
\text { N (\%) }\end{array}$ & $\begin{array}{c}\text { Exaustão } \\
\text { N (\%) }\end{array}$ & Total N (\%) & Valor de $\mathrm{p}^{*}$ \\
\hline Formação no Ensino Médio & & & & & & $=949$ \\
\hline Ens. Público & $19(25,68)$ & $39(52,7)$ & $12(16,22)$ & $4(5,41)$ & $74(100,0)$ & \\
\hline Ens. Privado & $2(33,33)$ & $4(66,67)$ & $0(0,0)$ & $0(0,0)$ & $6(100,0)$ & \\
\hline Total & $21(26,25)$ & $43(53,75)$ & $12(15,0)$ & $4(5,0)$ & $80(100,0)$ & \\
\hline Cotas & & & & & & $=0,949$ \\
\hline Sim & $6(27,27)$ & $11(50,0)$ & $4(18,18)$ & $1(4,55)$ & $22(100,0)$ & \\
\hline Não & $15(25,86)$ & $32(55,17)$ & $8(13,79)$ & $3(5,17)$ & $58(100,0)$ & \\
\hline Total & $21(26,25)$ & $43(53,75)$ & $12(15,0)$ & $4(5,0)$ & $80(100,0)$ & \\
\hline Tipo de cota & & & & & & $=0,019$ \\
\hline Esc. Pública & $3(42,86)$ & $3(42,86)$ & $0(0,0)$ & $1(14,29)$ & $7(100,0)$ & \\
\hline
\end{tabular}




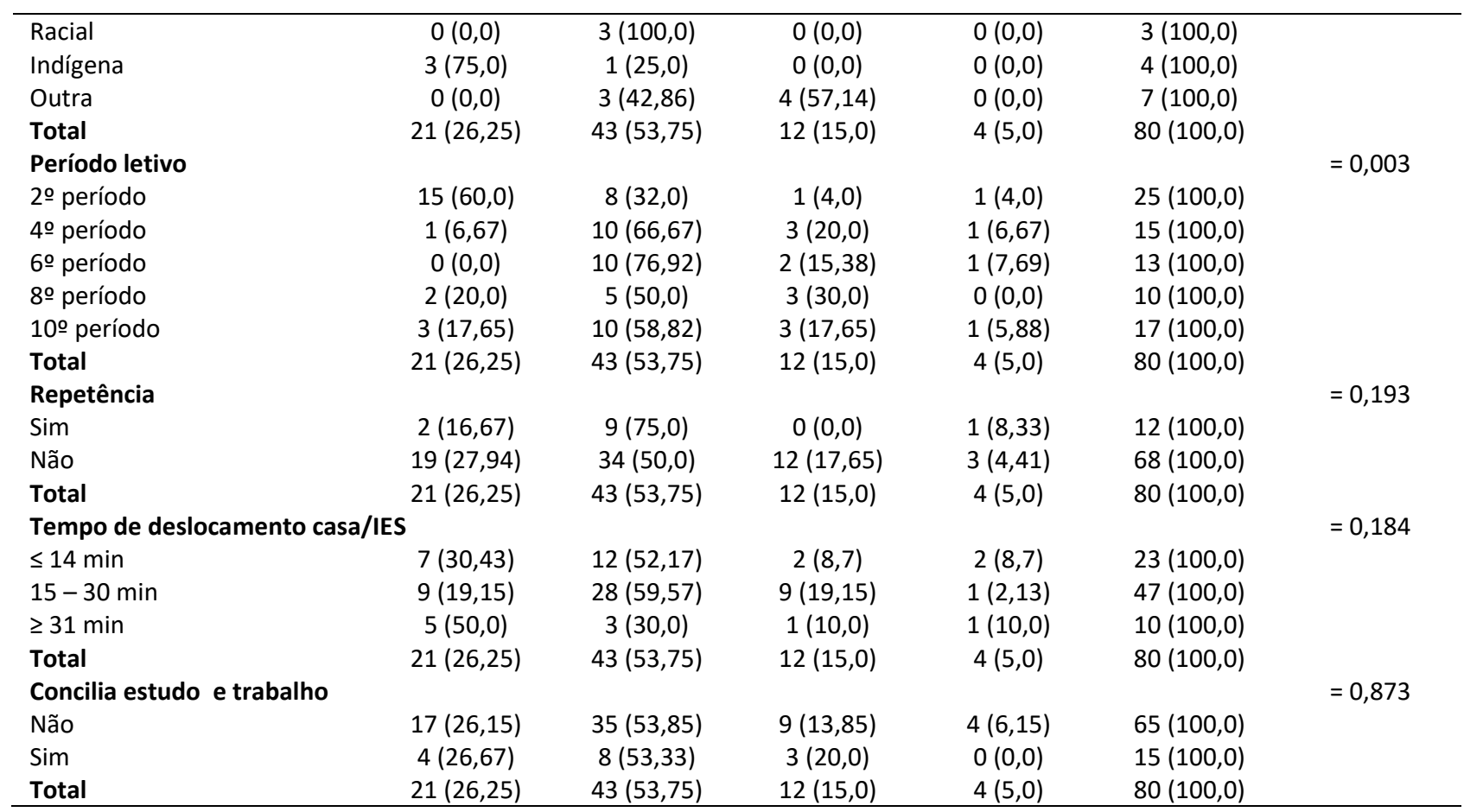

*Teste exato de Fisher: $p<0,05$.

A associação do desfecho com a variável "tipo de cota" pode estar relacionada ao rendimento acadêmico, menor entre alunos cotistas do que entre alunos não cotistas em cursos da área da saúde. A baixa qualidade do ensino público, a precariedade socioeconômica da maioria desses alunos, que se traduz na necessidade de trabalhar para ajudar a família, e a oferta insuficiente de bolsas de estudo tornam desafiadora a obrigação de atender às demandas acadêmicas e a responsabilidade de se manter na universidade $^{(19)}$, o que pode se configurar como estressores adicionais a esses alunos.

Ao analisar o nível de estresse por período acadêmico cursado, observam-se resultados mais significativos no sexto e no quarto período. Esses resultados podem ter relação com duas características específicas à grade curricular do curso em questão. Uma delas diz respeito às diferenças concretas de atividades em relação à grade curricular dos semestres anteriores, visto que o curso iniciou, em 2013, um movimento de mudança no seu processo de formação, passando a adotar uma estrutura de currículo integrado, desenvolvido em núcleos. Para a sua operacionalização, a organização do conhecimento se dá por saberes e componentes curriculares distribuídos ao longo de cinco anos, mas que somente a partir do quarto período, ocorre efetivamente a integração deles.

Nesse modelo, além das aulas expositivas e práticas, desenvolvem-se as atividades educativas a partir de dois cenários pedagógicos: a Situação Problema, que tem como pressuposto a Aprendizagem Baseada em Problemas (ABP), na qual os estudantes trabalham com o objetivo de solucionar um problema simulado a partir de um contexto $^{(20)}$ e Síntese oral decorrente das discussões de textos previamente selecionados.

A sistematização e a síntese do conhecimento produzido nos componentes curriculares ocorrem a partir da elaboração de portfólios que compõem as dimensões avaliativas. Nesse contexto, o quarto período marca bruscamente essa transição curricular que, somado ao início das atividades práticas em ambientes de saúde, podem ser fatores desencadeadores de estresse.

O estresse está presente em todos os períodos de formação, porém, tendem a se intensificar com o início das atividades práticas ${ }^{(21)}$. É nesse momento que ocorre o contato mais próximo com os pacientes e espera-se que o acadêmico demonstre habilidades práticas associadas aos conhecimentos teóricos. Com a inserção acadêmica no campo prático, situações como o medo de adquirir infecção, a sobrecarga de trabalho acadêmico, a dificuldade de comunicação e o contato com pacientes em estado grave e/ou em óbito têm-se constituído fontes de estresse adicionais aos fatores acadêmicos ${ }^{(22)}$.

De acordo com a literatura, quanto mais avançado o período em que o aluno se encontra, 
maior seu nível de estresse, significando que a preocupação com o conhecimento adquirido e seu impacto em sua futura vida profissional gera estresse cada vez maior à medida que o acadêmico avança no curso. Ademais, a natural preocupação com o desenvolvimento da carreira e da vida pessoal é um fator adicional à sua ocorrência ${ }^{(9,23)}$.

Nos anos iniciais, o acadêmico ainda não tem condições de elaborar o que significa estar no ensino superior que, geralmente, é visto como um período de idealização. Conforme vai avançando no curso, o acadêmico vai desenvolvendo a criticidade em relação a vários aspectos institucionais, estrutura, serviços, compromissos, entre outros. Ademais, a proximidade com a transição entre vida acadêmica e profissional pode se refletir em malestar físico e psicológico ${ }^{(18)}$.

$\mathrm{Na}$ avaliação de nível de estresse entre acadêmicos que precisam conciliar os estudos com o trabalho, evidenciou-se que os que não trabalham apresentaram níveis de estresse superiores aos que trabalham, o que se contrapõe ao que diz a literatura. Em relação a esses dados, em pesquisa realizada numa faculdade privada do interior de São Paulo, ficou evidente que a duplicidade de papéis é um fator que pode desencadear estresse.

A dificuldade de conciliar o trabalho e os estudos pode levar a um desgaste físico e psicológico, pois exige muito esforço e gasto de energia ${ }^{(3)}$. Considera-se que essa variável precisa ser explorada com mais afinco, em estudos subsequentes, para a devida compreensão do fenômeno.

A variável independente "uso de medicamentos" mostrou-se associada ao desfecho $(p=0,04)$ e do total de alunos pesquisados, $23,7 \%$ declararam utilizar medicamentos antidepressivos ou ansiolíticos. Desses, 89,5\% encontravam-se em algum nível de estresse. Entre os acadêmicos que declararam não fazer uso de medicamentos, o nível de estresse foi identificado em $68,8 \%$. Entre estes resultados, destaca-se a proporção de estudantes em nível de exaustão que declararam fazer uso de medicação, conforme Tabela 3.

Tabela 3 - Níveis de estresse conforme utilização de medicamentos por alunos de enfermagem de um Instituto Federal de Ensino, segundo ISSL. Brasil, out. 2016.

\begin{tabular}{|c|c|c|c|c|c|c|}
\hline \multicolumn{7}{|c|}{ Fases do Estresse } \\
\hline & Sem estresse & Resistência & $\begin{array}{c}\text { Quase } \\
\text { exaustão }\end{array}$ & Exaustão & & \\
\hline Variável & N (\%) & N (\%) & N (\%) & N (\%) & Total N (\%) & Valor de $p$ \\
\hline Uso de Medicamento & & & & & & $=0,04$ \\
\hline Não & $19(31,15)$ & $31(50,82)$ & $10(16,39)$ & $1(1,64)$ & $61(100,0)$ & \\
\hline Sim & $2(10,53)$ & $12(63,16)$ & $2(10,53)$ & $3(15,79)$ & $19(100,0)$ & \\
\hline Total & $21(26,25)$ & $43(53,75)$ & $12(15,0)$ & $4(5,0)$ & $80(100,0)$ & \\
\hline
\end{tabular}

*Teste exato de Fisher: $p<0,05$.

Acadêmicos que fazem uso de medicação de uso contínuo apresentaram maior tendência $(p=0,04)$ de apresentar sintomas de estresse e, entre eles, predominaram os sintomas correspondentes às fases de resistência e da quase exaustão, o que é um fator preocupante, uma vez que acadêmicos de enfermagem apresentam melhor desempenho em nível baixo de ansiedade ${ }^{(24)}$.

Corroborando com esses resultados, estudo desenvolvido em uma universidade pública do Estado de São Paulo, com o objetivo de avaliar a presença da ansiedade em estudantes de enfermagem, constatou que estudantes que estavam utilizando ansiolíticos no período em que a pesquisa foi realizada ou já haviam utilizado em algum momento de suas vidas apresentaram nível elevado de ansiedade ${ }^{(25)}$. Frente a esses resultados, destacase a importância de investigar a indicação do tratamento, bem como o conhecimento desses alunos sobre o uso e os efeitos da medicação para melhor esclarecer a manutenção dos sintomas.

Destarte, ressalta-se a importância de acompanhamento psicológico desses alunos com a finalidade de identificação de formas alternativas de diminuição do estresse, uma vez que o uso regular desses medicamentos oferece vários riscos aos usuários, tais como confusão mental, dificuldade de concentração e de memorização, problemas de ansiedade e de sono, além de sintomas de depressão, o que pode comprometer ainda mais 0 desempenho acadêmico. Ademais, quando não ocorre o acompanhamento profissional adequado, há o 
risco do abandono do tratamento, aumento da dose sem orientação médica, além de uso concomitante da medicação com bebida alcoólica $^{(25)}$.

Quanto à sintomatologia apresentada, predominaram os sintomas de caráter psicológico, sendo apresentados por $50 \%$ dos acadêmicos que apresentam algum nível de estresse, com destaque para a fase de resistência. É importante destacar, nessa associação, que os sintomas psicológicos tiveram maior ocorrência nos níveis de quase exaustão e exaustão. (Tabela 4).

Tabela 4 - Sintomatologia conforme fase do estresse dos acadêmicos de enfermagem de um Instituto Federal de Ensino, segundo ISSL. Brasil, out. 2016.

\begin{tabular}{lcccccc}
\hline & Sem estresse & Resistência & $\begin{array}{c}\text { Fases do Estresse } \\
\text { Quase } \\
\text { exaustão }\end{array}$ & Exaustão & & \\
Variável & $\mathbf{N}(\%)$ & $\mathbf{N}(\%)$ & $\mathbf{N}(\%)$ & $\mathbf{N}(\%)$ & Total N (\%) & Valor de $\mathbf{p} *$ \\
Sintoma & & & & & $<0,001$ \\
Ausente & $20(100,0)$ & $0(0,0)$ & $0(0,0)$ & $0(0,0)$ & $20(100,0)$ & \\
Físicos & $0(0,0)$ & $17(89,47)$ & $2(10,53)$ & $0(0,0)$ & $19(100,0)$ \\
Psicológicos & $0(0,0)$ & $18(60,0)$ & $8(26,67)$ & $4(13,33)$ & $30(100,0)$ \\
Físicos e Psicológicos & $1(9,09)$ & $8(72,73)$ & $2(18,18)$ & $0(0,0)$ & $11(100,0)$ \\
Total & $21(26,25)$ & $43(53,75)$ & $12(15,0)$ & $4(5,0)$ & $80(100,0)$ \\
\hline
\end{tabular}

*Teste exato de Fisher: $p<0,05$.

Esses resultados assemelham-se aos evidenciados por outros estudos em que os sintomas psicológicos também foram os mais presentes entre adultos jovens e acadêmicos de enfermagem com quadro de estresse ${ }^{(9-10)}$.

Em relação aos achados acima, os autores listam como possíveis fatores causadores de estresse nos alunos de enfermagem: conteúdo programático das atividades acadêmicas teóricas e práticas ${ }^{(2-3,7,9,14)}$, elaboração do trabalho de conclusão de curso $^{(3,9)}$, preocupações relacionadas à inserção no mercado de trabalho ${ }^{(3,9,14,22)}$, relação trabalho/estudo/vida familiar ${ }^{(3,9,14,22)}$, dificuldade no relacionamento interpessoal ${ }^{(3,7,9,22)}$, avaliações ${ }^{(7,9,14)}$, atividades extracurriculares ${ }^{(9)}$, falta de tempo para o lazer, família, para os amigos e para as necessidades pessoais ${ }^{(2,14)}$.

Considerando os efeitos do estresse na saúde física, mental e no rendimento acadêmico, recomenda-se o apoio psicológico aos discentes que vivenciam esse momento, no sentido de proporcionar segurança, desenvolver estratégias de enfrentamento dos fatores estressantes, bem como contribuir para o desenvolvimento de mecanismos que colaborem para um melhor aproveitamento do curso $^{(3)}$. Outro aspecto a se considerar no cenário estudado diz respeito à necessidade de inserção gradual dos acadêmicos na nova metodologia, de modo a minimizar os impactos na vida acadêmica.

\section{CONCLUSÃO}

O estudo revelou que a atividade acadêmica pode ser fonte de estresse, ao caracterizar $73,7 \%$ dos alunos em alguma fase desse estado, sendo a de resistência, a de maior predominância. Quanto à sintomatologia mais apresentada pela população do estudo, a psicológica foi a predominante.

Todos os períodos do curso de graduação em enfermagem apresentaram níveis preocupantes de estresse; porém, esses variam de acordo com o período do curso em que estão matriculados. As variáveis associadas ao desfecho foram "tipo de cota", "período letivo" e "uso de medicamentos". Quanto às maiores frequências de estresse, destacaram-se as ocorrências em indivíduos de sexo masculino, discentes que moram em pensão ou sozinhos e indivíduos que fazem uso de medicamentos contínuos, entre eles antidepressivos e ansiolíticos.

Cabe ressaltar que não era objetivo da presente pesquisa avaliar os fatores relacionados à manutenção do quadro disfuncional dos discentes que, por diferentes motivos, fazem uso de medicação antidepressiva e ansiolítica, como por exemplo; em que estágio do tratamento o indivíduo se encontra; se o acompanhamento está sendo realizado por médico especialista; adequação do medicamento e da dosagem, dentre outros. Salienta-se também a importância, diante dos transtornos emocionais, do acompanhamento psicoterápico aliado ao medicamentoso, por ser, a psicoterapia, um meio 
que possibilita, ao sujeito, um aprendizado de como lidar de forma mais assertiva com suas fraquezas, conflitos e emoções.

Devido à multicausalidade do estresse, não se pode afirmar que os resultados aqui obtidos estejam relacionados às questões acadêmicas; contudo, esse estudo pode ser considerado um passo inicial para a investigação de estresse entre o meio acadêmico de outros cursos do ensino superior, pesquisando sua ocorrência, sintomatologia, causas, entre outros fatores associados.

Entende-se que novas pesquisas em relação ao estresse no meio acadêmico poderão contribuir para que coordenações das instituições de ensino superior revejam suas metodologias de ensino e proponham ações que visem o bemestar acadêmico, tendo em vista a diminuição do estresse.

Na continuidade desse estudo, pretende-se investigar as causas dos estressores nessa população, como forma de subsidiar a Instituição de Ensino no desenvolvimento de estratégias de intervenção, de prevenção e de promoção de saúde, melhorando a qualidade de vida dos discentes e, consequentemente, melhorar o aproveitamento acadêmico e reduzir a evasão universitária.

Como limitação, cita-se a impossibilidade de generalizar os resultados da pesquisa, devido ao número reduzido de sujeitos do estudo por uma população específica de estudantes de uma instituição federal de ensino. Todavia, destacamos que a categorização em níveis da variável "desfecho para fins de análise", possibilitou a avaliação do gradiente de resposta para cada categoria das variáveis independentes analisadas.

\section{REFERÊNCIAS}

1. Lipp MEN. Stress: Conceitos básicos. In: Lipp MEN (Ed). Pesquisas sobre stress no Brasil: Saúde, ocupações e grupos de risco. Campinas: Papirus; 1996.

2. Lameu JN, Salazar TL, Souza WF de. Prevalência de sintomas de stress entre graduandos de uma universidade pública. Psicol Educ 2016;42:13-22. DOI: $10.5935 / 2175-3520.20150021$

3. Oliveira LA, Ferreira JS, Godinho RLP, Alves EA, Santos PSSR dos, Passos JP. Estresse nos acadêmicos de enfermagem de uma universidade pública. Arq Ciênc Saúde 2014 [citado em 6 jan 2018]; 21(2):118-23. Disponível em: http://repositorio-racs.famerp.br/racs ol/vol-212/ID 612 alter 21(2) Abr-jun 2014.pdf

4. Paraná. Secretaria de Educação Superior. Projeto Pedagógico do Curso de Enfermagem em processo para o reconhecimento. Paraná: Seaes; 2014.

5. Selye $H$. The stress of life. New York: Mc Graw Hill; 1956.

6. Lipp MEN. Manual do Inventário de Sintomas de Stress para Adultos de Lipp (ISSL). 3a ed. São Paulo: Casa do Psicólogo; 2015.

7. Benavante $S B T$, Silva RM, Higashi $A B$, Guido $A$, Costa ALS. Influência de fatores de stresse e características sociodemográficas na qualidade de sono dos estudantes de Enfermagem. Rev Esc Enferm USP 2014;48(3):514-20. DOI: 10.1590/50080-623420140000300018

8. Moura IH, Nobre RS, Cortez RMA, Campelo V, Macedo SF, Silva ARV. Qualidade de vida de estudantes de graduação em enfermagem. Rev Gaúcha Enferm. [internet]. 2016;37(2):1-7. DOI: http://dx.doi.org/10.1590/19831447.2016.02.552 $\underline{91}$

9. Mota NIF, Alves ERP, Leite GO, Sousa SMA, Ferreira Filha MO, Dias MD. Estresse entre graduandos de enfermagem de uma universidade pública. Rev Eletrônica Saúde Mental Álcool Drog. 2016;12(3):163-70. DOI: 10.11606/issn.18066976.v12i3p163-170

10. Fiorin PC, Oliveira CT de, Dias ACG. Percepções de mulheres sobre a relação entre trabalho e maternidade. Rev Bras Orientac Prof. 2014;15(1):25-35. [citado 201806 jan]. DOI: 10.11606/issn.1806-6976.v12i3p163-170

11. Instituto Brasileiro de Geografia e Estatística (IBGE). Estatísticas do registro civil. Estat Reg Civ. 2016;42:1-6.

12. Brasil. Instituto Brasileiro de Geografia e Estatística (IBGE). Pesquisa Nacional por amostra de domicílios: Síntese de indicadores 2014. Rio de Janeiro: IBGE; 2015.

13. Brasil. Ministério da Educação e Cultura (MEC). Balanço da política de quotas 2012-2013. Brasília: MEC; 2014.

14. Vilela SC, Pacheco AE, Carlos ALS. Síndrome de Burnout e estresse em graduandos de enfermagem. Rev Enferm Cent-Oeste Min. 2013;3(3): 780-7. DOI: 10.19175/recom.v0i0.415

15. Santos VEP, Radünz V. O estresse de acadêmicas de enfermagem e a segurança do paciente. Rev Enferm UERJ 2011 [citado em 22 ago 2016]; 19(4):616-20. Disponível em: http://www.facenf.uerj.br/v19n4/v19n4a19.pdf 
16. Moreira DP, Furegato ARF. Estresse e depressão entre alunos do último período de dois cursos de enfermagem. Rev Latino-Am Enfermagem 2013;21(nesp):1-8. DOI: 10.1590/S0104-11692013000700020

17. Friedrichi ACD, Macedo $F$, Reis $A H$. Vulnerabilidade ao stress em adultos jovens. Rev Psicol, Organ Trab. 2015;15(1):59-70. DOI: 10.17652/rpot/2015.1.499

18. Carlotto RC, Teixeira MAP, Dias ACG. Adaptação acadêmica e coping em estudantes universitários. Psico-USF 2015;20(3):421-32. DOI: 10.1590/1413-82712015200305

19. Peixoto ALA, Ribeiro EMBA, Bastos AVB, Ramalho MCK. Cotas e desempenho acadêmico na UFBA: um estudo a partir dos coeficientes de rendimento. Avaliação 2016;21(2):569-91. DOI: 10.1590/S1414-40772016000200013

20. Barros HSA. A taxonomy of problem-based learning methods. Med Educ. 1986 [citado em 8 jun 2018]; 20(6):481-6. Disponível em: https://onlinelibrary.wiley.com/doi/pdf/10.1111/ j.1365-2923.1986.tb01386.x

21. Pereira FGF, Caldini LN, Miranda MC, Caetano JA. Assessment of stress in the inclusion of nursing students in hospital practice. Invest Educ Enferm. 2014;32(3):430-7. DOI: 10.1590/S012053072014000300008

22. Rodriguez EOL, Marques DA, Lopes Neto D, Montesinos MJL, Oliveira ASA de. Stressful situations and factors in students of nursing in clinical practice. Invest Educ Enferm. 2016;34(1): 211-20. DOI: 10.17533/udea.iee.v34n1a23

23. Soares $\mathrm{MH}$, Oliveira FS. A relação entre álcool, tabaco e estresse em estudantes de Enfermagem. Rev Eletrônica Saúde Mental Álcool Drog. 2013;9(2):88-94. DOI: $10.11606 /$ issn.18066976.v9i2p88-94

24. Marchi KC, Bárbaro AM, Miasso Al, Tirapelli CR. Ansiedade e consumo de ansiolíticos entre estudantes de enfermagem de uma universidade pública. Rev Eletr Enf. 2013;15(3):731-9. DOI: 10.5216/ree.v15i3.1892

25. Brasil. Secretaria Nacional de Políticas sobre Droga. Efeitos de substâncias psicoativas: Módulo 2. 7a ed. Brasília: MJC; 2014.
Nota: Este manuscrito trata-se de um trabalho de conclusão de curso oriundo do Projeto de Pesquisa intitulado "Estresse no cotidiano acadêmico: com a voz discentes e docentes de um Instituto Federal de Ensino", desenvolvido no Instituto Federal do Paraná.

Recebido em: 17/01/2018

Aprovado em: 16/07/2018

Endereço de correspondência:

Leni de Lima Santana

R. João Negrão, 1285 - Rebouças

CEP: 80230-150 - Curitiba/PR - Brasil

E- mail: leni.santana@ifpr.edu.br 